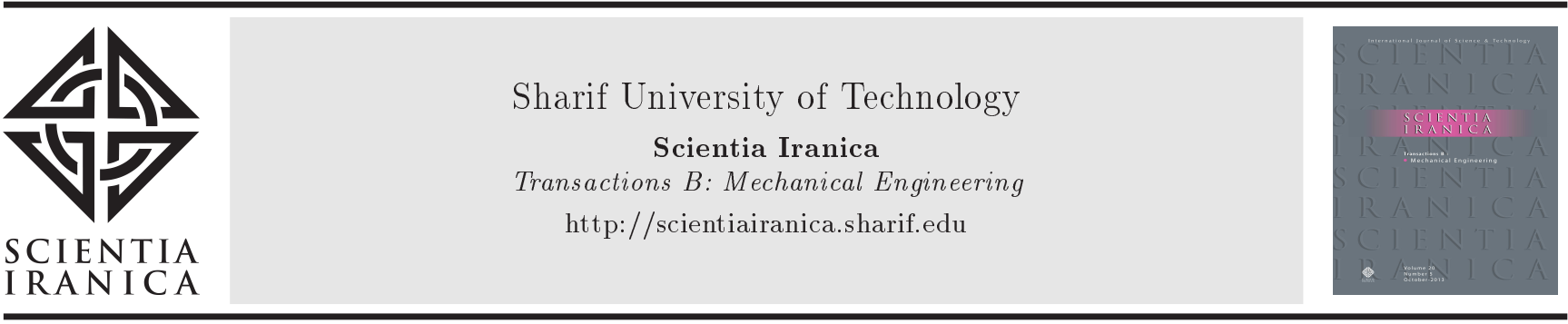

\title{
An explicit solution for the size-dependent large amplitude transverse vibration of thin functionally graded micro-plates
}

\author{
A.R. Setoodeh* and M. Rezaei \\ Department of Mechanical and Aerospace Engineering, Shiraz University of Technology, Shiraz 71555, Iran.
}

Received 18 November 2016; received in revised form 18 January 2017; accepted 4 March 2017

\author{
KEYWORDS \\ Functionally graded \\ micro-plates; \\ Nonlinear vibration; \\ Homotopy analysis \\ method; \\ Modified couple stress \\ theory; \\ Size effects.
}

\begin{abstract}
In this article, an analytical solution to the moderately large amplitude transverse vibration of thin Functionally Graded Micro-Plates (FGMPs) is presented based on a practical approach. The size-dependent nonlinear governing equation is obtained in conjunction with the Kirchhoff's plate and modified couple stress theories. The material properties of Functionally Graded (FG) micro-plates vary according to the Reddy's model. The employed non-classical theory contains one material length-scale parameter to capture the size effects. The highly nonlinear governing equation is solved by means of homotopy analysis method so as to obtain accurate analytic approximations. Both of simply supported and clamped micro-plates with immovable edges are considered. The comparison made between the present results and those of earlier studies confirms the reliability and effectiveness of the present formulation for the design purpose. Furthermore, the effects of different parameters, such as material gradient index, length-scale parameter, and aspect ratio, on the nonlinear frequency ratio are investigated.
\end{abstract}

(C) 2018 Sharif University of Technology. All rights reserved.

\section{Introduction}

Functionally Graded Materials (FGMs) are a kind of inhomogeneous composites usually made from a mixture of two materials. The properties vary with a smooth and continuous pattern from one surface to another as a function of position along a certain axis. This effectively eliminates the stress concentration found in laminated composites. In recent years, the application of FGMs has spread rapidly for micro- and nano-scale devices such as thin films in the form of shape memory alloys, atomic force microscopes, and Micro- and Nano-Electro-Mechanical Systems (MEMS

\footnotetext{
*. Corresponding author. Tel/Fax.: +98 7137264102 E-mail addresses: setoodeh@sutech.ac.ir and asetood@yahoo.com (A.R. Setoodeh)
}

and NEMS) [1]. Also, the two-dimensional structures with the order of microns or sub-microns have been widely used in MEMS, NEMS, and many other micro-structures. The properties of such elements are closely related to their microstructures. Thus, it is necessary to investigate the microstructure effects on the mechanical behavior of the aforementioned structures. The local continuum theories could not capture the micro-sized effects due to the lack of a material length-scale parameter; thus, non-classical theories, such as the couple stress, nonlocal elasticity, and strain gradient theories, have been proposed and widely implemented [2-13]. The strain gradient theory is a more general form of the higher-order non-classical theories taking into consideration both anti-symmetric and symmetric parts of the higher-order deformation gradients. However, this theory may involve some difficulties; therefore, alternative theories have been developed to effectively capture the size effect. 
Among higher-order continuum theories, the classical couple stress theory was introduced and extended by Toupin [14] as well as Mindlin and Tiersten [15]. They used two constants in order to take into account the size effects for isotropic materials. Meanwhile, Yang et al. [16] utilized the idea of the couple stress theory and proposed the modified version of the couple stress theory. The Modified Couple Stress Theory (MCST) benefits from two main advantages of the inclusion of symmetric couple stress tensor and involvement of only one material length-scale parameter. Based on the modified couple stress theory, different studies have been conducted concerning vibrational behavior of micro-plates [17-22].

Meanwhile, researchers have started to explore potential application of Functionally Graded (FG) microstructures by focusing on the vibration response analysis. Shenas et al. [23] investigated vibration of functionally graded micro beams with variable section in thermal environment. Therefore, in another work, Shenas et al. [24] presented vibrational behaviors of rotating pre-twisted functionally graded micro beams in thermal environment. Setoodeh and Rezaei [25] employed modified couple stress theory to analyze large amplitude free vibration of FG nano/micro beams on nonlinear elastic foundation. Ke and Wang [26] investigated size effects on the dynamic stability of functionally graded micro beams based on modified coupe stress theory. Setoodeh and Afrahim [27] developed a strain gradient model for nonlinear free vibration of FG micro pipes conveying fluid. Thai and Kim [28] developed a size-dependent model for bending and free vibration of functionally graded Reddy plate using analytical solutions. Thai and Choi [29] presented a model with one material length-scale parameter for bending, buckling, and vibration of FG Kirchhoff and Mindlin plates. Thai and Vo [30] developed a sizedependent model for bending and free vibration of FG plates based on the MCST and sinusoidal shear deformation theory.

Although micro plates are susceptible to large amplitude vibration on the basis of design consideration, only few studies have explored nonlinear vibration behavior of FG micro-plates. In this regard, Ke et al. [31] presented a non-classical model for axisymmetric nonlinear free vibration analysis of annular micro plates made of FGMs using MCST. Therefore, Ansari et al. [32] investigated nonlinear vibrations of FG Mindlin micro plates based on the modified couple stress theory. Lou and He [33] provided closed-form solutions for nonlinear bending and free vibration of functionally graded micro plates based on the modified couple stress theory using physical neutral surface. Mohammadimehr and Mohandes [34] investigated the effect of modified couple stress theory on buckling and vibration analysis of functionally graded double-layer piezoelectric plates. He et al. [35] presented a sizedependent four-variable refined plate model for functionally graded micro-plates based on modified couple stress theory. Shenas and Malekzadeh [36] investigated free vibration of functionally graded quadrilateral micro plates in thermal environment. Thus, according to the available literature, no analytical expressions for nonlinear frequencies of FG micro plates have been derived so far. Normally, it is desirable for designers to provide explicit expressions for linear and nonlinear fundamental frequencies in conformity with a reasonable engineering approximation, whenever it is possible. In this regard, the Homotopy Analysis Method (HAM) has been successfully implemented already on a wide range of nonlinear vibration problems [3739]. The HAM possesses certain superiority compared with the conventional perturbation methods. The perturbation methods for any perturbation quantity and the modified iteration method can be considered as the special case of the HAM [40].

The structure of this article is organized as follows: Section 2 presents the displacement field and material properties of FG micro plates. In Section 3, the nonlinear dynamic governing equation of functionally graded thin micro-plates is derived in the context of the modified couple stress theory using Hamilton's principle. The governing equation is obtained in terms of the stress resultants, and the related boundary conditions are introduced. In Section 4, firstly, the Galerkin method is used to solve the resulted nonlinear partial differential equation; afterwards, the governing equation is analytically solved using homotopy analysis method. In addition, the analytical explicit expressions for the size-dependent linear and nonlinear natural frequencies of the FG micro plates are presented. The accuracy of the explicit solution is illustrated in the subsequent section, wherein the model is used to carry out some parametric studies. The last section closes this article with a brief conclusion.

\section{Displacement field and material properties}

Figure 1 shows a rectangular FGMP with in-plane dimensions $a$ and $b$ in $x$ and $y$ directions, respectively,

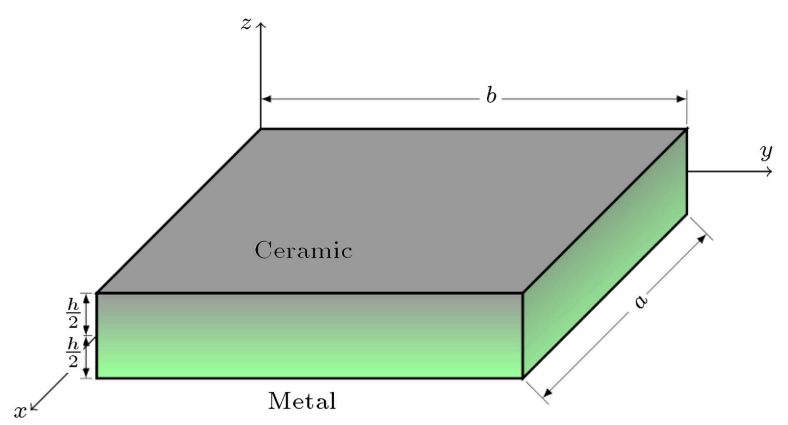

Figure 1. Schematic representation of a FG micro-plate. 
and thickness $h$ in $z$ direction. The reference Cartesian coordinate system $(x, y, z)$ is located on the mid-plane of the plate. The FGMP is made of a mixture of ceramic and metal. The lower surface $(z=-h / 2)$ and upper surface $(z=h / 2)$ are pure metal and ceramic, respectively. In this study, the material properties are assumed to vary in terms of power law distribution given by:

$$
p(z)=p_{m}+\left(p_{c}-p_{m}\right) V_{c},
$$

where $p_{c}$ and $p_{m}$ are, respectively, the corresponding properties of ceramic $(c)$ and metal $(m)$, and $V_{c}=$ $(z / h+0.5)^{n}$ is the volume fraction of ceramic. Also, $n$ denotes the material gradient index which takes values greater than or equal to zero. The volume fractions of ceramic and metal phases are defined as in $V_{c}+V_{m}=1$. Among the properties, the Poisson's ratio is assumed to be constant in the numerical results.

The displacement components $\left(u_{x}, u_{y}, u_{z}\right)$ along $(x, y, z)$ directions are given according to the Kirchhoff's plate theory in terms of mid-surface displacements $\left(u_{0}, \nu_{0}\right)$ and transverse deflection $w$ as follows:

$$
\begin{aligned}
& u_{x}(x, y, z, t)=u_{0}-z \frac{\partial w(x, y, t)}{\partial x}, \\
& u_{y}(x, y, z, t)=\nu_{0}-z \frac{\partial w(x, y, t)}{\partial y}, \\
& u_{z}(x, y, z, t)=w(x, y, t) .
\end{aligned}
$$

\section{The modified couple stress governing equation}

According to the modified couple stress theory, strain energy, $U$, for a deformable linear elastic material that occupies volume, $V$, can be written as follows [4]:

$$
U=\frac{1}{2} \int_{V}\left(\sigma_{i j} \varepsilon_{i j}+m_{i j} \chi_{i j}\right) d V, \quad(i, j=x, y, z)
$$

where $\sigma_{i j}, \varepsilon_{i j}, m_{i j}$, and $\chi_{i j}$ are, respectively, stress tensor, strain tensor, deviatoric part of the couple stress tensor, and symmetric curvature tensor defined as follows:

$$
\begin{aligned}
& \sigma_{i j}=\lambda \delta_{i j} \varepsilon_{k k}+2 \mu \varepsilon_{i j}, \\
& \lambda=\frac{E \nu}{(1+\nu)(1-2 \nu)}, \quad \mu=\frac{E}{2(1+\nu)}, \\
& \chi_{i j}=\frac{1}{2}\left(\theta_{i, j}+\theta_{j, i}\right), \\
& m_{i j}=2 l^{2} \mu \chi_{i j},
\end{aligned}
$$

where, in all relations $(i, j=x, y, z), \lambda$ and $\mu$ are the Lame's constants, $E$ and $\nu$ are, respectively, Young's modulus and Poisson's ratio, and $l$ denotes the material length-scale parameter. Also, $u_{i}$ represents the components of displacement vector, $\mathbf{u}$, and $\theta_{i}$ refers to the components of rotation vector defined as follows:

$$
\theta_{i}=\frac{1}{2}[\operatorname{curl}(u)]_{i} .
$$

The Green strain-displacement relation, which takes into account the nonlinear terms, is as follows:

$$
\varepsilon_{i j}=\frac{1}{2}\left(u_{i, j}+u_{j, i}+u_{k, i} u_{k, j}\right), \quad i, j, k=x, y, z .
$$

Using Eqs. (2) and (8) for a thin elastic plate, based on the von Karman kinematic assumptions, the strain components in terms of the displacements can be written as follows:

$$
\begin{aligned}
& \varepsilon_{x x}=\frac{\partial u_{0}}{\partial x}+\frac{1}{2}\left(\frac{\partial w}{\partial x}\right)^{2}-z \frac{\partial^{2} w}{\partial x^{2}}, \\
& \varepsilon_{y y}=\frac{\partial \nu_{0}}{\partial y}+\frac{1}{2}\left(\frac{\partial w}{\partial y}\right)^{2}-z \frac{\partial^{2} w}{\partial y^{2}}, \\
& \gamma_{x y}=\frac{\partial u_{0}}{\partial y}+\frac{\partial \nu_{0}}{\partial x}+\frac{\partial w}{\partial x} \frac{\partial w}{\partial y}-2 z \frac{\partial^{2} w}{\partial x \partial y}, \\
& \gamma_{x z}=\gamma_{y z}=\varepsilon_{z z}=0 .
\end{aligned}
$$

For a thin elastic plate with moderately large amplitude vibration in the absence of in-plane forces and assuming that the edges of the plate are immovable, the nonzero stress components can be found in terms of displacement components according to the linear elastic constitutive relations as follows [41-44]:

$$
\begin{aligned}
\sigma_{x x}= & \frac{E(z)}{1-\nu^{2}(z)}\left\{-z \frac{\partial^{2} w}{\partial x^{2}}+\frac{1}{2}\left(\frac{\partial w}{\partial x}\right)^{2}\right. \\
& \left.+\nu(z)\left[\frac{1}{2}\left(\frac{\partial w}{\partial y}\right)^{2}-z \frac{\partial^{2} w}{\partial y^{2}}\right]\right\}, \\
\sigma_{y y}= & \frac{E(z)}{1-\nu^{2}(z)}\left\{-z \frac{\partial^{2} w}{\partial y^{2}}+\frac{1}{2}\left(\frac{\partial w}{\partial y}\right)^{2}\right. \\
+ & \left.\nu(z)\left[\frac{1}{2}\left(\frac{\partial w}{\partial x}\right)^{2}-z \frac{\partial^{2} w}{\partial x^{2}}\right]\right\}, \\
\sigma_{x y}= & \frac{E(z)}{2(1+\nu(z))}\left(\frac{\partial w}{\partial x} \frac{\partial w}{\partial y}-2 z \frac{\partial^{2} w}{\partial x \partial y}\right) .
\end{aligned}
$$

Eq. (5) in conjunction with Eq. (7) results in the components of curvature tensor as follow:

$$
\chi_{x x}=\frac{\partial^{2} w}{\partial x \partial y}, \chi_{y y}=-\frac{\partial^{2} w}{\partial x \partial y}, \chi_{x y}=\frac{1}{2}\left(\frac{\partial^{2} w}{\partial y^{2}}-\frac{\partial^{2} w}{\partial x^{2}}\right)
$$

Subsequently, the components of the couple stress 
tensor are found by substituting Eq. (11) into Eq. (6) as follows:

$$
\begin{aligned}
& m_{x x}=2 l^{2} \mu(z) \chi_{x x}=l^{2} \frac{E(z)}{1+\nu(z)} \frac{\partial^{2} w}{\partial x \partial y} \\
& m_{y y}=2 l^{2} \mu(z) \chi_{y y}=-l^{2} \frac{E(z)}{1+\nu(z)} \frac{\partial^{2} w}{\partial x \partial y} \\
& m_{x y}=2 l^{2} \mu(z) \chi_{x y}=\frac{1}{2} l^{2} \frac{E(z)}{1+\nu(z)}\left(\frac{\partial^{2} w}{\partial y^{2}}-\frac{\partial^{2} w}{\partial x^{2}}\right) \\
& m_{x z}=m_{y z}=m_{z z}=0 .
\end{aligned}
$$

By expanding Eq. (3), the variation of strain energy of the FGMP can be written as follows:

$$
\begin{aligned}
\delta U= & \int_{A} \int_{-h / 2}^{h / 2}\left(\sigma_{x x} \delta \varepsilon_{x x}+\sigma_{y y} \delta \varepsilon_{y y}+\sigma_{x y} \delta \gamma_{x y}\right. \\
& \left.+m_{x x} \delta \chi_{x x}+m_{y y} \delta \chi_{y y}+2 m_{x y} \delta \chi_{x y}\right) d z d A \\
= & \int_{A}\left[N_{x x} \frac{\partial w}{\partial x} \frac{\partial \delta w}{\partial x}-M_{x x} \frac{\partial^{2} \delta w}{\partial x^{2}}+N_{y y} \frac{\partial w}{\partial y} \frac{\partial \delta w}{\partial y}\right. \\
& -M_{y y} \frac{\partial^{2} \delta w}{\partial y^{2}}-2 M_{x y} \frac{\partial^{2} \delta w}{\partial x \partial y}+N_{x y}\left(\frac{\partial w}{\partial y} \frac{\partial \delta w}{\partial x}\right. \\
& \left.+\frac{\partial w}{\partial x} \frac{\partial \delta w}{\partial y}\right)+P_{x x} \frac{\partial^{2} \delta w}{\partial x \partial y}-P_{y y} \frac{\partial^{2} \delta w}{\partial x \partial y} \\
& \left.+P_{x y}\left(\frac{\partial^{2} \delta w}{\partial y^{2}}-\frac{\partial^{2} \delta w}{\partial x^{2}}\right)\right] d A
\end{aligned}
$$

where $A$ is the cross-sectional area of the micro-plate. Also, the stress resultants are defined as follows:

$$
\begin{aligned}
& \left(N_{\alpha \beta}, M_{\alpha \beta}\right)=\int_{-h / 2}^{h / 2}(1, z) \sigma_{\alpha \beta} d z, \quad(\alpha, \beta=x, y), \\
& P_{\alpha \beta}=\int_{-h / 2}^{h / 2} m_{\alpha \beta} d z, \quad(\alpha \beta=x, y) .
\end{aligned}
$$

The kinetic energy and variation of kinetic energy of the FGMP are defined as follows:

$$
\begin{aligned}
T=\frac{1}{2} \int_{V} \rho \dot{u}_{i} \dot{u}_{i} d V=\frac{1}{2} \int_{A} \int_{-h / 2}^{h / 2} \rho\left(\left(\frac{\partial u_{x}}{\partial t}\right)^{2}\right. \\
\left.+\left(\frac{\partial u_{y}}{\partial t}\right)^{2}+\left(\frac{\partial u_{z}}{\partial t}\right)^{2}\right) d z d A, \quad(i=x, y, z),(16) \\
\delta T=\int_{A}\left[I_{0} \frac{\partial w}{\partial t} \frac{\partial \delta w}{\partial t}+I_{2}\left(\frac{\partial \dot{w}}{\partial x} \frac{\partial \delta \dot{w}}{\partial x}+\frac{\partial \dot{w}}{\partial y} \frac{\partial \delta \dot{w}}{\partial y}\right)\right] d A,
\end{aligned}
$$

where dot overscript convention denotes the differentiation with respect to time variable, $t$. Also, various inertias are calculated according to the power law distribution of properties as follows:

$$
\begin{aligned}
\left(I_{0}, I_{2}\right)= & \int_{-h / 2}^{h / 2}\left[\left(\rho_{m}\left(1, z^{2}\right)\right)+\left(\rho_{c}-\rho_{m}\right)\left(\frac{z}{h}\right.\right. \\
& \left.+0.5)^{n}\left(1, z^{2}\right)\right] d z .
\end{aligned}
$$

The nonlinear dynamic governing equations of the FG micro plate are derived using the Hamilton's principle which is stated in terms of the variation of strain energy $(\delta U)$, variation of kinetic energy $(\delta T)$, and variation of the work done by external forces $(\delta W)$ :

$$
\int_{t_{1}}^{t_{2}}[\delta(T-U)+\delta W] d t=0
$$

In the absence of body moments and surface tractions and using the fundamental lemma of the calculus of variation, the governing equation in terms of the stress resultants is obtained following some mathematical manipulations:

$$
\begin{aligned}
\frac{\partial^{2} M_{x x}}{\partial x^{2}} & +2 \frac{\partial^{2} M_{x y}}{\partial x \partial y}+\frac{\partial^{2} M_{y y}}{\partial y^{2}}+\frac{\partial^{2} P_{x y}}{\partial x^{2}}+\frac{\partial^{2} P_{y y}}{\partial x \partial y} \\
& -\frac{\partial^{2} P_{x y}}{\partial y^{2}}-\frac{\partial^{2} P_{x x}}{\partial x \partial y}+N(w) \\
& =I_{0} \ddot{w}-I_{2}\left(\frac{\partial^{2} \ddot{w}}{\partial x^{2}}+\frac{\partial^{2} \ddot{w}}{\partial y^{2}}\right)
\end{aligned}
$$

The stress resultants can also be expanded in terms of displacements' components as follows:

$$
\begin{aligned}
M_{x x}= & \int_{-h / 2}^{h / 2} \sigma_{x x} z d z=-C_{2} \frac{\partial^{2} w}{\partial x^{2}}+\frac{B_{2}}{2}\left(\frac{\partial w}{\partial x}\right)^{2} \\
& +\frac{B_{3}}{2}\left(\frac{\partial w}{\partial y}\right)^{2}-C_{3} \frac{\partial^{2} w}{\partial y^{2}} \\
M_{y y}= & \int_{-h / 2}^{h / 2} \sigma_{y y} z d z=-C_{2} \frac{\partial^{2} w}{\partial y^{2}}+\frac{B_{2}}{2}\left(\frac{\partial w}{\partial y}\right)^{2} \\
& +\frac{B_{3}}{2}\left(\frac{\partial w}{\partial x}\right)^{2}-C_{3} \frac{\partial^{2} w}{\partial x^{2}} \\
M_{x y}= & \int_{-h / 2}^{h / 2} \sigma_{x y} z d z=\frac{B_{1}}{2} \frac{\partial w}{\partial x} \frac{\partial w}{\partial y}-C_{1} \frac{\partial^{2} w}{\partial x \partial y}
\end{aligned}
$$




$$
\begin{aligned}
N_{x x}= & \int_{-h / 2}^{h / 2} \sigma_{x x} d z=-B_{2} \frac{\partial^{2} w}{\partial x^{2}}+\frac{A_{2}}{2}\left(\frac{\partial w}{\partial x}\right)^{2} \\
& +\frac{A_{3}}{2}\left(\frac{\partial w}{\partial y}\right)^{2}-B_{3} \frac{\partial^{2} w}{\partial y^{2}} \\
N_{y y}= & \int_{-h / 2}^{h / 2} \sigma_{y y} d z=-B_{2} \frac{\partial^{2} w}{\partial y^{2}}+\frac{A_{2}}{2}\left(\frac{\partial w}{\partial y}\right)^{2} \\
& +\frac{A_{3}}{2}\left(\frac{\partial w}{\partial x}\right)^{2}-B_{3} \frac{\partial^{2} w}{\partial x^{2}}, \\
N_{x y}= & \int_{-h / 2}^{h / 2} \sigma_{x y} d z=\frac{A_{1}}{2} \frac{\partial w}{\partial x} \frac{\partial w}{\partial y}-B_{1} \frac{\partial^{2} w}{\partial x \partial y} \\
P_{x x}= & \int_{-h / 2}^{h / 2} m_{x x} d z=A_{1} l^{2} \frac{\partial^{2} w}{\partial x \partial y}, \\
P_{x y}= & \int_{-h / 2}^{h / 2} m_{x y} d z=\frac{A_{1}}{2} l^{2}\left(\frac{\partial^{2} w}{\partial y^{2}}-\frac{\partial^{2} w}{\partial x^{2}}\right) \\
P_{y y}= & \int_{-h / 2}^{h / 2} m_{y y} d z=-A_{1} l^{2} \frac{\partial^{2} w}{\partial x \partial y}, \\
&
\end{aligned}
$$

where:

$$
\begin{aligned}
& \left\{A_{1}, B_{1}, C_{1}\right\}=\int_{-h / 2}^{h / 2} \frac{E(z)}{1+\nu(z)}\left\{1, z, z^{2}\right\} d z \\
& \left\{A_{2}, B_{2}, C_{2}\right\}=\int_{-h / 2}^{h / 2} \frac{E(z)}{1-\nu^{2}(z)}\left\{1, z, z^{2}\right\} d z \\
& \left\{A_{3}, B_{3}, C_{3}\right\}=\int_{-h / 2}^{h / 2} \frac{E(z) \nu(z)}{1-\nu^{2}(z)}\left\{1, z, z^{2}\right\} d z .
\end{aligned}
$$

Also, $N(w)$ is defined by:

$$
\begin{aligned}
N(w)= & \frac{\partial}{\partial x}\left(N_{x x} \frac{\partial w}{\partial x}+N_{x y} \frac{\partial w}{\partial y}\right) \\
& +\frac{\partial}{\partial y}\left(N_{x y} \frac{\partial w}{\partial x}+N_{y y} \frac{\partial w}{\partial y}\right) .
\end{aligned}
$$

It should be noted that if length-scale parameter, $l$, is set to zero, the equation of motion is reduced to the form of the classical continuum theory.

The boundary conditions of the following types are considered for FGMPs:

(a) Fully simply supported:

$$
w(0, y, t)=w(a, y, t)=w(x, 0, t)=w(x, b, t)=0
$$

$$
\begin{gathered}
\frac{\partial^{2} w(0, y, t)}{\partial x^{2}}=\frac{\partial^{2} w(a, y, t)}{\partial x^{2}}=\frac{\partial^{2} w(x, 0, t)}{\partial y^{2}} \\
=\frac{\partial^{2} w(x, b, t)}{\partial y^{2}}=0 .
\end{gathered}
$$

(b) Fully clamped:

$$
\begin{gathered}
w(0, y, t)=w(a, y, t)=w(x, 0, t)=w(x, b, t)=0 \\
\frac{\partial w(0, y, t)}{\partial x}=\frac{\partial w(a, y, t)}{\partial x}=\frac{\partial w(x, 0, t)}{\partial y}= \\
\frac{\partial w(x, b, t)}{\partial y}=0
\end{gathered}
$$

\section{Method of solution}

The techniques of separation of variables in conjunction with the Galerkin method are used to solve the resulted nonlinear partial differential equation. The transverse displacement can be written as follows:

$$
w(x, y, t)=W(t) X(x) Y(y) .
$$

Based on the first mode shape of vibration, functions $X(x)$ and $Y(y)$ might be defined according to Eqs. (29) and (30) for fully simply supported and fully clamped end conditions, respectively [45]:

$$
\begin{aligned}
& X(x)= \sin \left(\frac{\pi x}{a}\right), \quad Y(y)=\sin \left(\frac{\pi y}{b}\right) \\
& X(x)=c\left[\cosh \left(\frac{q x}{a}\right)-\cos \left(\frac{q x}{a}\right)-\frac{\cosh (q)-\cos (q)}{\sinh (q)-\sin (q)}\right. \\
&\left.\left(\sinh \left(\frac{q x}{a}\right)-\sin \left(\frac{q x}{a}\right)\right)\right] \\
& Y(y)= {\left[\cosh \left(\frac{q y}{b}\right)-\cos \left(\frac{q y}{b}\right)-\frac{\cosh (q)-\cos (q)}{\sinh (q)-\sin (q)}\right.} \\
&\left.\left(\sinh \left(\frac{q y}{b}\right)-\sin \left(\frac{q y}{b}\right)\right)\right] \\
& c=0.6297, q=4.730041
\end{aligned}
$$

By substituting Eq. (28) into Eq. (20), multiplying the result by functions, $X(x) Y(y)$, and then integrating them over the area of the plate, a new form of the governing equation is obtained as follows:

$$
\frac{\partial^{2} W}{\partial t^{2}}+\bar{\alpha} W+\bar{\beta} W^{2}+\bar{\gamma} W^{3}=0
$$

The coefficients in Eq. (31) are defined as shown in the Appendix.

\subsection{Homotopy analysis method}

The developed nonlinear governing differential equation is analytically solved for two different boundary 
conditions using homotopy analysis method. The HAM initially introduced by Liao [46] is a powerful and computationally cost-effective method which is capable of solving strongly nonlinear differential equations. At first, the solution procedure is briefly explained. For further details, one can refer to [46]. Consider a series of time-dependent nonlinear differential equations as follows:

$$
N_{i}\left[z_{i}(t)\right]=0 \quad i=1,2, \ldots, n .
$$

In Eq. (32), $N_{i}$ represents nonlinear operators, $t$ denotes an independent variable, and $z_{i}(t)$ represents unknown functions. Liao [46] constructed the so-called zero-order deformation equations as follows:

$$
(1-q) L\left[\phi_{i}(t ; q)-z_{i, 0}(t)\right]=q \hbar_{i} h_{i}(t) N_{i}\left[\phi_{i}(t ; q)\right]
$$

where $q$ is an embedding parameter that varies in the range of $[0,1], \hbar_{i}$ represents nonzero auxiliary parameters, and $h_{i}(t)$ denotes nonzero auxiliary functions. $z_{i, 0}(t)$ represents the initial guesses of $z_{i}(t), \phi_{i}(t ; q)$ represents unknown functions, and $L$ is the selected auxiliary linear operator. There are some degrees of freedom to choose auxiliary linear operator and $h(t)$. Parameters $\hbar_{i}$ and $h_{i}(t)$ adjust the convergence region of the solution. Here, when $q$ increases from 0 to 1 , the solutions $\phi_{i}(t, q)$ alter from the initial guesses to $z_{i}(t)$ solutions. In other words, $\phi_{i}$ takes the following forms for $q=0$ and $q=1$, respectively:

$$
\phi_{i}(t ; 0)=z_{i, 0}(t), \quad \phi_{i}(t ; 1)=z_{i}(t)
$$

By differentiating Eq. (33) with respect to $q$, the firstorder deformation equation can be obtained as follows:

$$
L\left[z_{i, 1}(t)\right]=\hbar_{i} h_{i}(t) N_{i}\left[\phi_{i}(t ; q)\right]_{q=0} .
$$

With expanding $\phi_{i}(t ; q)$ in the form of Taylor series, one obtains:

$$
\phi_{i}(t ; q)=z_{i, 0}(t)+\sum_{m=1}^{+\infty} z_{i, m}(x, t) q^{m}
$$

where:

$$
z_{i, m}=\left.\frac{1}{m !} \frac{\partial^{m} \phi_{i}(t ; q)}{\partial q^{m}}\right|_{q=0}
$$

By substituting Eq. (36) into the constructed zeroorder equation and differentiating the resulted equation $m$ times with respect to embedding parameter $q$, then setting $q=0$, and finally dividing all of the terms by $m$ !, the so-called $m$ th-order deformation equation is obtained as follows:

$$
L\left[z_{i, m}(t)-\chi_{m} z_{i, m-1}(t)\right]=\hbar_{i} h(t) R_{i, m}\left(\vec{z}_{i, m-1}\right),
$$

where:

$$
\begin{gathered}
\vec{z}_{i, m}=\left\{z_{i, 0}(t), z_{i, 1}(t), \ldots, z_{i, m}(t)\right\} \\
R_{i, m}\left(\vec{z}_{i, m-1}\right)=\left.\frac{1}{(m-1) !} \frac{\partial^{m-1} N_{i}\left[\phi_{i}(t ; q)\right]}{\partial q^{m-1}}\right|_{q=0} \\
\chi_{m}=\left\{\begin{array}{l}
0, m \leq 1, \\
0, m>1 .
\end{array}\right.
\end{gathered}
$$

\subsection{Explicit expressions for nonlinear frequencies}

It is convenient to transform Eq. (31) by setting a new variable $\tau=\omega t$, where $\omega$ denotes the nonlinear frequency. After some manipulations, one obtains:

$$
\omega^{2} \frac{d^{2} V(\tau)}{d \tau^{2}}+\bar{\alpha} V(\tau)+\bar{\beta} V^{2}(\tau)+\bar{\gamma} V^{3}(\tau)=0
$$

subject to the following initial conditions at the center of the micro-plate:

$$
V_{0}(0)=W_{\max }, \quad \frac{d V_{0}(0)}{d \tau}=0 .
$$

Herein, $W_{\max }$ is the maximum amplitude of the FGMP vibration. Initial guess, $V_{0}(\tau)$, should be selected, such that the initial conditions are satisfied:

$$
V_{0}(\tau)=W_{\max } \cos (\tau)
$$

The linear and nonlinear operators can be written as follows:

$$
\begin{aligned}
L[\phi(\tau ; q)]=\omega_{0}^{2}( & \left.\frac{d^{2} \phi(\tau ; q)}{d \tau^{2}}+\phi(\tau ; q)\right), \\
N[\phi(\tau ; q), \omega(q)]= & \omega^{2}(q) \frac{d^{2} \phi(\tau ; q)}{d \tau^{2}}+\bar{\alpha} \phi(\tau ; q) \\
& +\bar{\beta} \phi^{2}(\tau ; q)+\bar{\gamma} \phi^{3}(\tau ; q) .
\end{aligned}
$$

In Eq. (45), $\omega(q)$ is defined as follows:

$$
\omega(q)=\omega_{0}+\omega_{1} q+\omega_{2} q^{2}+\ldots
$$

In order for the present solution to achieve accurate results by only few terms, we set $h(t)=1$, and $\bar{h}=-1$. Also, to start the solution procedure, parameter $m$ is considered to be 1 in Eq. (38), which is read as follows: 


$$
\begin{aligned}
R_{1}\left(z_{0}(t)\right)= & N[\phi(t ; 0)] \\
= & \omega_{0}{ }^{2} \frac{d^{2} \phi(\tau ; 0)}{d \tau^{2}}+\bar{\alpha} \phi(\tau ; 0)+\bar{\beta} \phi^{2}(\tau ; 0) \\
& +\bar{\gamma} \phi^{3}(\tau ; 0) \\
= & \omega_{0}{ }^{2} \frac{d^{2} V_{0}(\tau)}{d \tau^{2}}+\bar{\alpha} V_{0}(\tau)+\bar{\beta} V_{0}^{2}(\tau) \\
& +\bar{\gamma} V_{0}{ }^{3}(\tau)
\end{aligned}
$$

Subsequently, the first-order deformation equation is found according to Eq. (35) as follows:

$$
\begin{gathered}
\omega_{0}^{2}\left(\frac{d^{2} V_{1}(\tau)}{d \tau^{2}}+V_{1}(\tau)\right)=\left(\omega_{0}^{2} \frac{d^{2} V_{0}(\tau)}{d \tau^{2}}\right. \\
\left.+\bar{\alpha} V_{0}(\tau)+\bar{\beta} V_{0}^{2}(\tau)+\bar{\gamma} V_{0}^{3}(\tau)\right)
\end{gathered}
$$

where the initial conditions are:

$$
V_{1}(0)=\frac{d V_{1}(0)}{d \tau}=0
$$

After substituting $V_{0}(\tau)$ from Eq. (43) into Eq. (48) and solving the resulted equation, one obtains:

$$
\begin{aligned}
V_{1}(\tau) & =\frac{2 \bar{\beta} W_{\max }^{2}}{3 \omega_{0}^{2}}+\left(\frac{\left(4 \bar{\alpha}-4 \omega_{0}^{2}\right) W_{\max }+3 \bar{\gamma} W_{\max }^{3}}{8 \omega_{0}^{2}}\right) \\
& \tau \sin (\tau)+\left(\frac{-8 \bar{\beta} W_{\max }^{2}+3 \bar{\gamma} W_{\max }^{3}}{24 \omega_{0}^{2}}\right) \cos (\tau) \\
& -\left(\frac{\bar{\beta} W_{\max }^{2}}{3 \omega_{0}^{2}}\right) \cos ^{2}(\tau)-\left(\frac{\bar{\gamma} W_{\max }^{3}}{8 \omega_{0}^{2}}\right) \cos ^{3}(\tau) .
\end{aligned}
$$

The coefficient of the secular term, $\tau \sin (\tau)$, must be zero since the amplitude of the micro-plate vibration is finite. Thus:

$$
\bar{\gamma} W_{\max }^{2}-\frac{4}{3} \omega_{0}^{2}+\frac{4}{3} \bar{\alpha}=0 .
$$

The solution of Eq. (51) leads to the first approximation of the nonlinear frequency $\left(\omega_{0}\right)$ as follows:

$$
\omega_{0}=\sqrt{\bar{\alpha}+\frac{3}{4} \bar{\gamma} W_{\max }^{2}}
$$

Now, $m$ is set to 2 in order to obtain $V_{2}(\tau)$. In view of Eqs. (38), (40), (44), (45), and (50), the second stage of formulations can be written as follows:

$$
L\left[V_{2}(\tau)-V_{1}(\tau)\right]=\left.\hbar h(t) \frac{\partial N[\phi(t ; q)]}{\partial q}\right|_{q=0}
$$

$$
\begin{aligned}
& \omega_{0}^{2}\left(\frac{d^{2} V_{2}(\tau)}{d \tau^{2}}+V_{2}(\tau)\right)-\omega_{0}^{2}\left(\frac{d^{2} V_{1}(\tau)}{d \tau^{2}}+V_{1}(\tau)\right) \\
& =\left[2 \omega(q) \frac{d \omega(q)}{d q} \frac{\partial^{2} \phi(\tau ; q)}{\partial \tau^{2}}+\omega(q)^{2} \frac{\partial^{2}}{\partial \tau^{2}}\left(\frac{\partial \phi(\tau ; q)}{\partial q}\right)\right. \\
& \quad+\alpha \frac{\partial \phi(\tau ; q)}{\partial q}+2 \beta \phi(\tau ; q) \frac{\partial \phi(\tau ; q)}{\partial q} \\
& \left.\quad+3 \gamma \phi^{2}(\tau ; q) \frac{\partial \phi(\tau ; q)}{\partial q}\right]_{q=0}
\end{aligned}
$$

Considering the initial conditions in Eq. (42):

$$
V_{2}(0)=\frac{d V_{2}(0)}{d \tau}=0 .
$$

Using the developed code, which is prepared in Maple software, $V_{2}(\tau)$ is obtained as follows:

$$
\begin{aligned}
V_{2}(\tau) & =\left(\frac{8}{15} A_{2}+\frac{2}{3} A_{4}+A_{6}\right)+\left(\frac{7}{48} A_{1}-\frac{1}{5} A_{2}\right. \\
& \left.+\frac{1}{8} A_{3}-\frac{1}{3} A_{4}-A_{6}\right) \cos (\tau) \\
& -\left(\frac{64 A_{2}+80 A_{4}}{240}\right) \cos ^{2}(\tau)-\left(\frac{25 A_{1}+30 A_{3}}{240}\right) \cos ^{3}(\tau) \\
& -\left(\frac{A_{2}}{15}\right) \cos ^{4}(\tau)-\left(\frac{A_{1}}{24}\right) \cos ^{5}(\tau) \\
& +\left(\frac{5 A_{1}+6 A_{3}+8 A_{5}}{16}\right) \tau \sin (\tau)
\end{aligned}
$$

where:

$$
\begin{aligned}
A_{1}= & -\frac{3}{8} \frac{\bar{\gamma}^{2} W_{\max }^{5}}{\omega_{0}^{4}}, \quad A_{2}=-\frac{5}{4} \frac{\bar{\gamma} \bar{\beta} W_{\max }^{4}}{\omega_{0}^{4}} \\
A_{3}= & \frac{\left(-3 \bar{\alpha} \bar{\gamma}-16 \bar{\beta}^{2}+51 \bar{\gamma} \omega_{0}^{2}\right) W_{\max }^{3}-24 \bar{\beta} \bar{\gamma} W_{\max }^{4}+9 \bar{\gamma}^{2} W_{\max }^{5}}{24 \omega_{0}^{4}} \\
A_{4}= & \frac{\left(56 \bar{\beta} \omega_{0}^{2}-8 \bar{\alpha} \bar{\beta}\right) W_{\max }^{2}-16 \bar{\beta}^{2} W_{\max }^{3}+54 \bar{\beta} \bar{\gamma} W_{\max }^{4}}{24 \omega_{0}^{4}} \\
A_{5}= & \left(\frac{4 \omega_{0} \omega_{1}-\bar{\alpha}-\omega_{0}^{2}}{2 \omega_{0}^{2}}\right) W_{\max }+\left(\frac{\bar{\beta} \omega_{0}^{2}-\bar{\alpha} \bar{\beta}}{3 \omega_{0}^{4}}\right) W_{\max }^{2} \\
& +\left(\frac{3 \bar{\alpha} \bar{\gamma}+32 \bar{\beta}^{2}-12 \bar{\gamma} \omega_{0}^{2}}{24 \omega_{0}^{4}}\right) W_{\max }^{3} \\
A_{6}= & \left(\frac{2 \bar{\alpha}-2 \bar{\beta} \omega_{0}^{2}}{3 \omega_{0}^{4}}\right) W_{\max }^{2} .
\end{aligned}
$$

Again, the coefficient of the secular term $\tau \sin (\tau)$ should be identical to zero. Therefore:

$$
A_{1}+\frac{6}{5} A_{3}+\frac{8}{5} A_{5}=0 .
$$


The second analytical approximation for the nonlinear frequency $\left(\omega_{1}\right)$ is achieved according to Eq. (58) by substituting the calculated coefficients $A_{1}, A_{3}$, and $A_{5}$ from Eq. (57) and solving the resulted equation:

$$
\omega_{1}=-\frac{160 \bar{\beta}^{2} W_{\max }^{2}-96 \bar{\gamma} \bar{\beta} W_{\max }^{3}+\frac{9}{2} \bar{\gamma}^{2} W_{\max }^{4}}{384\left(\bar{\alpha}+\frac{3}{4} \bar{\gamma} W_{\max }^{2}\right)\left(\sqrt{\bar{\alpha}+\frac{3}{4} \bar{\gamma} W_{\max }^{2}}\right)}
$$

Finally, the analytical expressions for the linear and nonlinear natural frequencies of FG micro plates are presented as follows:

$$
\begin{aligned}
\omega_{L}= & \sqrt{\bar{\alpha}} \\
\omega_{N L}= & \omega_{0}+\omega_{1}=\sqrt{\bar{\alpha}+\frac{3}{4} \bar{\gamma} W_{\max }^{2}} \\
& -\frac{160 \bar{\beta}^{2} W_{\max }^{2}-96 \bar{\gamma} \bar{\beta} W_{\max }^{3}+\frac{9}{2} \bar{\gamma}^{2} W_{\max }^{4}}{384\left(\bar{\alpha}+\frac{3}{4} \bar{\gamma} W_{\max }^{2}\right)\left(\sqrt{\bar{\alpha}+\frac{3}{4} \bar{\gamma} W_{\max }^{2}}\right)},
\end{aligned}
$$

where $\omega_{L}$ and $\omega_{N L}$ denote the linear and nonlinear natural frequencies, respectively. Also, $V(\tau)$ can be obtained by collecting the related terms as follows:

$$
V(\tau)=V_{0}(\tau)+V_{1}(\tau)+V_{2}(\tau) .
$$

\section{Numerical results}

Before proceeding with the parametric study of the large amplitude free flexural vibration behavior of FG micro-plates, the validity and accuracy of the formulation developed here are confirmed against the available results. Afterwards, further numerical results obtained through studying the size-dependent nonlinear vibration analysis of the FGMPs are presented using developed analytical expressions. FGMPs with two types of boundary conditions, including SSSS and CCCC, are considered, where simply supported and clamped edges are designated, respectively, by $\mathrm{S}$ and $\mathrm{C}$. In all of the developed results, the following dimensionless parameters are defined, unless otherwise specified.

$$
\eta_{\max }=\frac{W_{\max }}{h}, \quad \bar{\omega}_{L}=\frac{\omega_{L} a^{2}}{h} \sqrt{\frac{\rho_{c}}{E_{c}}}, \quad \Omega=\frac{\omega_{N L}}{\omega_{L}} .
$$

\subsection{Validation studies}

Table 1 lists the fundamental nonlinear frequency ratio of a clamped square isotropic plate in terms of dimensionless maximum amplitude, $\eta_{\max }$. The present solution is compared with the classical plate theory solution of Han and Petyt [47] which provided distinct results with and without inclusion of the in-plane displacements. The plate has a length of $0.5 \mathrm{~m}$, height of $2 \mathrm{~mm}$, and material properties of $E=210 \mathrm{GPa}$, $\nu=0.3$, and $\rho=7800 \mathrm{~kg} / \mathrm{m}^{3}$. It is noticeable that the present results agree well with the corresponding solution of [47] which takes into account the in-plane displacements. It is worth noting that this agreement holds over a wide range of the dimensionless maximum amplitude.

To better clarify the efficacy of the present formulation, nonlinear frequency ratios of simply supported FGM plates reported by Sundararajan et al. [48] are compared successfully with those obtained in this study as shown in Table 2. A moderately thick plate with a thickness ratio of $a / h=10$ and two gradient indices is considered. The solution of [48] is based on the Mindlin's plate theory. The FGM plate considered consists of silicon nitride $\left(\mathrm{Si}_{3} \mathrm{~N}_{4}\right)$ and stainless steel (SUS304) with material properties of $\rho_{c}=2370 \mathrm{~kg} / \mathrm{m}^{3}$, $\rho_{m}=8166 \mathrm{~kg} / \mathrm{m}^{3}, E_{c}=322.2715 \mathrm{GPa}, E_{m}=$ 207.7877 GPa, and $\nu=0.28$. Again, the present results agree well with those predicted by Sundararajan et al. [48]. In this example, the accuracy and reliability of the approach are effectively confirmed through the comparison carried out between the Mindlin's plate theory and a common value of the thickness ratio. To validate the size dependency of the natural frequency resulted from the present approach, some comparisons are made with other relevant solutions from the literature as shown in Table 3. Dimensionless linear natural frequencies, $\bar{\omega}$, of an isotropic square micro plate with simply supported boundary conditions are obtained in terms of the proposed admissible values of dimensionless length-scale parameter $(l / h)$ in $[19,28]$ and two different aspect ratios. An analytical solution was developed for vibration analysis of micro-scale plates based on the modified couple stress theory in the context of the Classical Plate Theory (CPT) in [19], while a sizedependent Navier solution was presented for bending and free vibration of functionally graded micro plates using the Third-order Shear Deformation Theory of Reddy (TSDT) [28]. Herein, the dimensionless natural

Table 1. Comparison of nonlinear frequency ratios, $\Omega$, of a clamped square isotropic plate with and without mid-plane displacements.

\begin{tabular}{cccc}
\hline \multirow{2}{*}{$\boldsymbol{\eta}_{\max }$} & $\begin{array}{c}\text { With mid-plane } \\
\text { displacements } \\
\text { Ref. [47] }\end{array}$ & \multicolumn{2}{c}{$\begin{array}{c}\text { Without mid-plane } \\
\text { displacements }\end{array}$} \\
\cline { 3 - 4 } & 1.0072 & 1.0082 & 1.0079 \\
0.2 & 1.0285 & 1.0324 & 1.0311 \\
0.4 & 1.0631 & 1.0714 & 1.0686 \\
0.6 & 1.1095 & 1.1235 & 1.1187 \\
0.8 & 1.1663 & 1.1866 & 1.1797 \\
0.1 & 1.2320 & 1.2591 & 1.2498 \\
1.2 & 1.3052 & 1.3392 & 1.3276 \\
1.4 & & &
\end{tabular}


Table 2. Comparison of nonlinear frequency ratios, $\Omega$, of simply supported FGM plates.

\begin{tabular}{|c|c|c|c|c|c|c|c|}
\hline \multirow{3}{*}{$a / h$} & \multirow{3}{*}{$n$} & \multicolumn{6}{|c|}{$\eta_{\max }$} \\
\hline & & \multicolumn{2}{|c|}{0.2} & \multicolumn{2}{|c|}{0.6} & \multicolumn{2}{|c|}{1} \\
\hline & & Ref. [48] & Present & Ref. [48] & Present & Ref. [48] & Present \\
\hline \multirow{2}{*}{10} & 2 & 1.0057 & 1.0268 & 1.1654 & 1.2108 & 1.4666 & 1.5251 \\
\hline & 10 & 1.0163 & 1.0262 & 1.1905 & 1.2136 & 1.4963 & 1.5164 \\
\hline
\end{tabular}

Table 3. Comparison of dimensionless linear natural frequencies for an isotropic simply supported square micro-plate.

\begin{tabular}{ccccc}
\hline \multirow{2}{*}{$/ \boldsymbol{h}$} & $\boldsymbol{l} / \boldsymbol{h}$ & \multicolumn{3}{c}{$\overline{\boldsymbol{\omega}}$} \\
\cline { 3 - 5 } & & Present & TSDT [28] & $\mathbf{C P T}[\mathbf{1 9}]$ \\
\hline & $\mathbf{0}$ & 5.9608 & 5.9199 & 5.9734 \\
$\mathbf{2 0}$ & $\mathbf{0 . 2}$ & 6.4420 & 6.4027 & 6.4556 \\
& $\mathbf{0 . 4}$ & 7.7076 & 7.6708 & 7.7239 \\
& $\mathbf{0 . 6}$ & 9.4474 & 9.4116 & 9.4673 \\
& $\mathbf{0 . 8}$ & 11.4470 & 11.4108 & 11.4713 \\
& $\mathbf{1}$ & 13.5926 & 13.5545 & 13.6213 \\
\hline & $\mathbf{0}$ & 5.9725 & 5.9712 & 5.9734 \\
& $\mathbf{0 . 2}$ & 6.4547 & 6.4535 & 6.4556 \\
$\mathbf{1 0 0}$ & $\mathbf{0 . 4}$ & 7.7228 & 7.7217 & 7.7239 \\
& $\mathbf{0 . 6}$ & 9.4660 & 9.4651 & 9.4673 \\
& $\mathbf{0 . 8}$ & 11.4697 & 11.4689 & 11.4713 \\
& $\mathbf{1}$ & 13.6194 & 13.6186 & 13.6213 \\
\hline
\end{tabular}

frequency is defined as $\bar{\omega}=\left(\omega a^{2} / h\right)(\rho / E)^{0.5}$. Also, the micro plate is made of epoxy with the material properties [28]:

$$
\begin{aligned}
& E=1.44 \mathrm{GPa} \quad \nu=0.3, \quad \rho=1220 \mathrm{~kg} / \mathrm{m}^{3}, \\
& h=88 \times 10^{-6} \mathrm{~m} .
\end{aligned}
$$

Again, it is observed that the present results are in close agreement with those reported in the aforementioned references; specifically, they are more accurate in comparison with those of the CPT solution. This demonstrates again the validity of the previous assumptions made in developing the governing equation of thin micro plates.

\subsection{Parametric studies}

The FGMP considered here is composed of aluminum (Al) and alumina $\left(\mathrm{Al}_{2} \mathrm{O}_{3}\right)$. The mechanical properties of the FGMP are listed as:

Metal (Al):

$$
E_{m}=70 \mathrm{GPa}, \quad \rho_{m}=2702 \mathrm{~kg} / \mathrm{m}^{3}, \quad \nu_{m}=0.3 ;
$$

Ceramic $\left(\mathrm{Al}_{2} \mathrm{O}_{3}\right)$ :

$$
E c=380 \mathrm{GPa}, \quad \rho_{c}=3800 \mathrm{~kg} / \mathrm{m}^{3}, \quad \nu_{c}=0.3 .
$$

Parametric studies are presented to investigate the effects of various variables such as dimensionless length scale parameter, $h / l$, dimensionless maximum amplitude, $\eta_{\max }$, material gradient index, $n$, aspect ratio, $a / b$, and boundary conditions on the nonlinear free vibration of the FGMPs using the developed analytical expressions. Initially, the value of material lengthscale parameter $l$ was proposed equal to $17.6 \mu \mathrm{m}$ based on the experimental work reported by Lam et al. [2] for homogeneous epoxy beams. However, so far, no experimental data have been available for the functionally graded micro plates [28]. Therefore, in this study, the length-scale parameter of the micro plate is approximately assumed to be $l=15 \mu \mathrm{m}$ wherever it is needed. Meanwhile, in order to present a comprehensive parametric study, the results are exhibited in terms of the dimensionless form of the length-scale parameter as $h / l$. It is worth noting that the influence of the length-scale parameter is only considerable when the thickness of plate is small and in order of micron scale, but the size effect is negligible when the plate thickness becomes larger [28].

In order to illustrate the importance of performing a nonlinear non-classical analysis for FGMPs, variations of different frequency ratios versus dimensionless length scale parameter, $h / l$, based on classical and nonclassical continuum theories are depicted in Figure 2. The results show the significant impact of length-scale parameter on the nonlinear vibration of FGMPs. It is also revealed that, only for the values of $h / l$ greater than 7 , one can ignore the size effects captured by the present nonlinear non-classical model.

Table 4 demonstrates the influences of dimensionless length-scale parameter on dimensionless linear frequency, $\bar{\omega}_{L}$, as well as the nonlinear frequency ratio for different boundary conditions. It is seen that with increasing the dimensionless length-scale parameter, the dimensionless linear frequency decreases, while the nonlinear frequency ratio increases. It can be further viewed that the nonlinear frequency ratio increases with the increase in the dimensionless maximum amplitude, as expected. 
Table 4. Effects of dimensionless length scale parameter $h / l$ on dimensionless linear frequency, $\bar{\omega}_{L}$, and nonlinear to linear frequency ratio, $\Omega$, for different boundary conditions $(n=2, a / b=1$, and $a / h=50)$.

\begin{tabular}{cccccc}
\hline \multirow{2}{*}{$\begin{array}{c}\text { Boundary } \\
\text { condition }\end{array}$} & $\boldsymbol{h} / \boldsymbol{l}$ & $\overline{\boldsymbol{\omega}}_{\boldsymbol{L}}$ & \multicolumn{3}{c}{$\boldsymbol{\eta}_{\max }$} \\
\cline { 4 - 6 } & & & $\mathbf{0 . 2}$ & $\mathbf{0 . 6}$ & $\mathbf{1}$ \\
\hline \multirow{2}{*}{ SSSS } & Classic & 4.7481 & 1.0247 & 1.2015 & 1.4897 \\
& $\mathbf{1 0}$ & 4.8364 & 1.0238 & 1.1949 & 1.4750 \\
& $\mathbf{6}$ & 4.9894 & 1.0224 & 1.1842 & 1.4511 \\
& $\mathbf{3}$ & 5.5619 & 1.0175 & 1.1465 & 1.3655 \\
& $\mathbf{2}$ & 6.6102 & 1.0128 & 1.1094 & 1.2786 \\
$\mathbf{C C C C}$ & $\mathbf{1}$ & 10.3510 & 1.0053 & 1.0463 & 1.1231 \\
\hline & $\mathbf{C l a s s i c}$ & 8.6854 & 1.0096 & 1.0828 & 1.2144 \\
& $\mathbf{1 0}$ & 8.8469 & 1.0093 & 1.0803 & 1.2082 \\
& $\mathbf{6}$ & 9.1269 & 1.0087 & 1.0754 & 1.1963 \\
& $\mathbf{2}$ & 10.3388 & 1.0068 & 1.0594 & 1.1563 \\
& $\mathbf{1}$ & 18.9346 & 1.0020 & 1.0182 & 1.0496 \\
\hline
\end{tabular}

Table 5. Effects of material gradient index, $n$, on the dimensionless linear frequency, $\bar{\omega}_{L}$, and nonlinear to linear frequency ratio, $\Omega$, for different boundary conditions $(h / l=2, a / b=1$, and $a / h=50)$.

\begin{tabular}{cccccc}
\hline $\begin{array}{c}\text { Boundary } \\
\text { condition }\end{array}$ & $\boldsymbol{n}$ & $\overline{\boldsymbol{\omega}}_{L}$ & \multicolumn{3}{c}{$\boldsymbol{\eta}_{\max }$} \\
\cline { 4 - 6 } & & & $\mathbf{0 . 2}$ & $\mathbf{0 . 6}$ & $\mathbf{1}$ \\
\hline \multirow{2}{*}{ SSSS } & $\mathbf{A l}_{\mathbf{2}} \mathbf{O}_{\mathbf{3}}$ & 8.5492 & 1.0136 & 1.1158 & 1.2940 \\
& $\mathbf{2}$ & 6.6102 & 1.0128 & 1.1094 & 1.2786 \\
& $\mathbf{1 0}$ & 5.4543 & 1.0117 & 1.1002 & 1.2567 \\
& $\mathbf{A l}$ & 3.1153 & 1.0264 & 1.2147 & 1.5190 \\
\hline \multirow{2}{*}{$\mathbf{C C C C}$} & $\mathbf{A l}_{\mathbf{2}} \mathbf{O}_{\mathbf{3}}$ & 15.6386 & 1.0053 & 1.0463 & 1.1234 \\
& $\mathbf{2}$ & 12.0916 & 1.0050 & 1.0438 & 1.1167 \\
& $\mathbf{1 0}$ & 9.9772 & 1.0045 & 1.0401 & 1.1071 \\
& $\mathbf{A l}$ & 5.6990 & 1.0103 & 1.0883 & 1.2282 \\
\hline
\end{tabular}

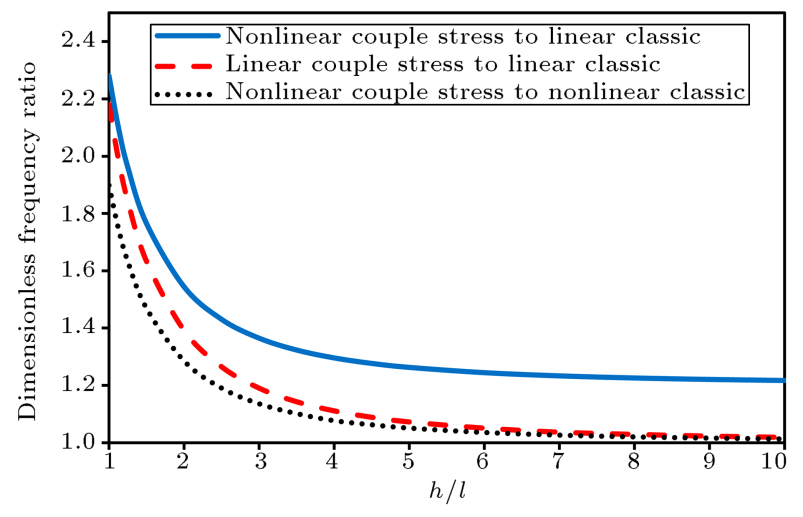

Figure 2. Frequency ratio versus dimensionless length scale parameter $h / l(n=2, a / b=1, a / h=50$, and $\left.\eta_{\max }=0.6\right)$.

Table 5 investigates the effects of material gradient index, $n$, on the dimensionless linear frequency and nonlinear frequency ratio of simply supported and clamped FGMPs. The dimensionless length-scale parameter is assumed to be identical to 2. In general, when $n$ increases from a zero value $\left(\mathrm{Al}_{2} \mathrm{O}_{3}\right)$ to infinity $(\mathrm{Al})$, the dimensionless linear frequency decreases. However, the non-dimensional nonlinear frequency ratio decreases with the increase in the material gradient index up to a particular value; afterwards, it increases with further increase in $n$. Such behavior can be seen in different analyses. In fact, for higher values of $n$, although the linear as well as nonlinear frequency values decrease, the reduction in linear frequency is more considerable in comparison to the corresponding value of the nonlinear frequency. Due to the greater reduction in the linear frequency at higher values of $n$, the trend of the resulted nonlinear frequency ratio is increasing.

In addition, the variations of non-dimensional nonlinear frequency ratio with respect to material property gradient index, $n$, and the maximum amplitude 


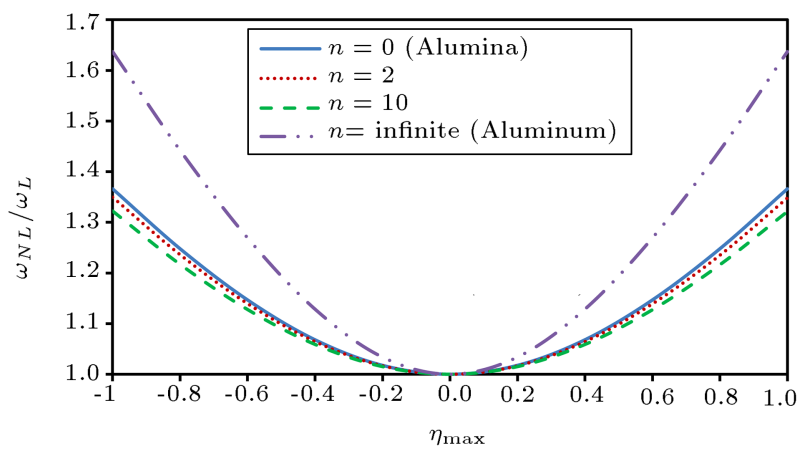

(a)

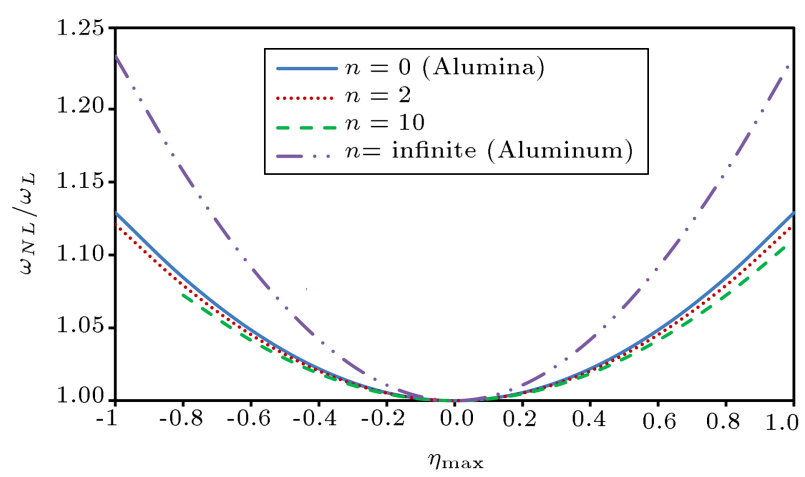

(b)

Figure 3. Effect of material property gradient index, $n$, on dimensionless frequency ratio versus dimensionless maximum amplitude for FGMPs $(h / l=2, a / b=2$, and $a / h=50):($ a) SSSS and (b) CCCC.

ratio are illustrated in Figure 3. The FGMP with both simply supported and clamped boundary conditions is considered.

Figure 4 demonstrates the variations of dimensionless frequency ratio with dimensionless maximum amplitude corresponding to different ratios of microplate aspect ratio. These variations are depicted for simply supported and clamped boundary conditions. It is found that the dimensionless frequency ratio increases with increasing the aspect ratio. However, this effect is enhanced for the case of simply supported FGMPs as the overall stiffness is decreased.

\section{Conclusions}

In this investigation, the size-dependent explicit expressions for the nonlinear vibration of thin FG micro plates are presented based on modified couple stress and Kirchhoff's plate theories. A power law distribution rule is used to capture the variation properties through the thickness of the plate. Hamilton's principle is employed to obtain the nonlinear governing equation of FGMPs. The homotopy analysis method in conjunction with the Galerkin technique is utilized to solve the resulted nonlinear differential equation. The analytical solutions are provided for simply supported

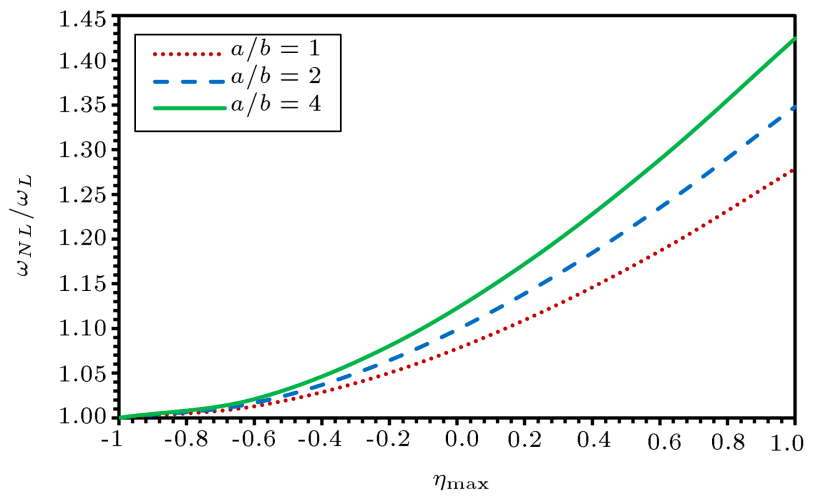

(a)

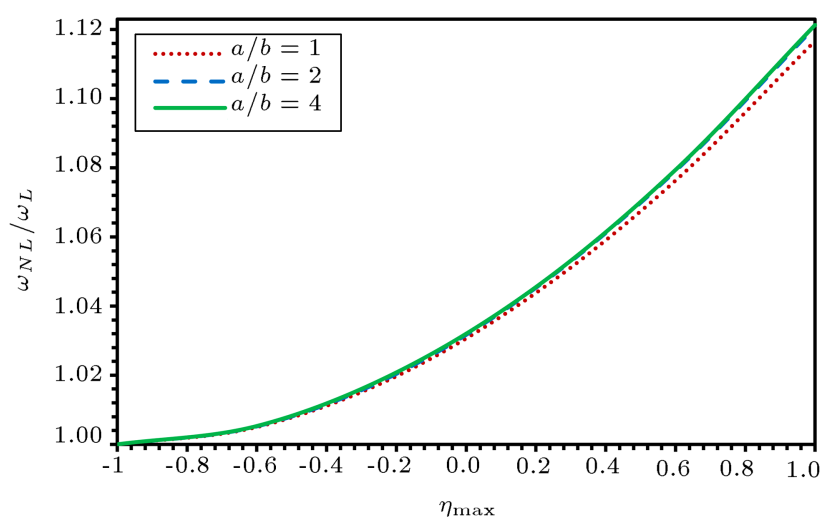

(b)

Figure 4. Effects of aspect ratio $a / b$ on dimensionless frequency ratio versus dimensionless maximum amplitude for FGMPs $(n=2, h / l=2$, and $a / h=50)$ : (a) SSSS and (b) CCCC.

and clamped FGMPs with immovable edges in the absence of in-plane forces. The efficacy and accuracy of the present formulation are tested by studying the nonlinear free flexural vibration of thin isotropic and FGM plates with simply supported and clamped boundary conditions for several examples, which are available in the literature. The model enables researchers to straightforwardly examine the effects of various parameters on the nonlinear frequencies. For instance, parameter studies are carried out to investigate the effects of different parameters, such as dimensionless length scale parameter, dimensionless maximum amplitude, material gradient index, aspect ratio, and boundary conditions, on the nonlinear frequencies of the FGMPs using the developed analytical expressions.

The numerical results reveal that the length-scale parameter significantly affects the nonlinear vibration of FGMPs. It is found that the stiffness of micro plates and, consequently, the frequency increase with the inclusion of the small-scale effects. It is also observed that the nonlinear natural frequencies of FGMPs deviate from the corresponding linear frequencies that exhibit the necessity of performing a nonlinear analysis for moderately large amplitude vibrations. 


\section{References}

1. Witvrouw, A., and Mehta, A. "The use of functionally graded poly-SiGe layers for MEMS applications", In Materials Science Forum. Trans. Tech. Publ., 492, pp. 255-260 (2005).

2. Lam, D.C.C., Yang, F., Chong, A.C.M., Wang, J., and Tong, P. "Experiments and theory in strain gradient elasticity", Journal of the Mechanics and Physics of Solids, 51, pp. 1477-1508 (2003).

3. Malekzadeh, P. and Shojaee, M. "A two-variable firstorder shear deformation theory coupled with surface and nonlocal effects for free vibration of nanoplates", Journal of Vibration and Control, 21, pp. 2755-2772 (2015).

4. Setoodeh, A.R., Rezaei, M., and Zendehdel Shahri, M.R. "Linear and nonlinear torsional free vibration of functionally graded micro/nano-tubes based on modified couple stress theory", Applied Mathematics and Mechanics (English Edition), 37, pp. 1-16 (2016).

5. Peng, X.W., Guo, X.M., Liu, L., and Wu, B.J. "Scale effects on nonlocal buckling analysis of bilayer composite plates under non-uniform uniaxial loads", Applied Mathematics and Mechanics (English Edition), 36, pp. 1-10 (2015).

6. Akgöz, B. and Civalek, Ö. "A size-dependent shear deformation beam model based on the strain gradient elasticity theory", International Journal of Engineering Science, 70, pp. 1-14 (2013).

7. Ma, H.M., Gao, X.L., and Reddy, J.N. "A non-classical Mindlin plate model based on a modified couple stress theory", Acta Mechanica, 220, pp. 217-235 (2011).

8. Gholami, R. and Ansari, R. "A most general strain gradient plate formulation for size-dependent geometrically nonlinear free vibration analysis of functionally graded shear deformable rectangular microplates", Nonlinear Dynamics, 84, pp. 2403-2422 (2016).

9. Akgoz, B. and Civalek, O. "A new trigonometric beam model for buckling of strain gradient microbeams", International Journal of Mechanical Sciences, 81, pp. 88-94 (2014).

10. Akgoz, B. and Civalek, O. "Bending analysis of FG microbeams resting on Winkler elastic foundation via strain gradient elasticity", Composite Structures, 134, pp. 294-301 (2015).

11. Akgoz, B. and Civalek, O. "microstructure-dependent sinusoidal plate model based on the strain gradient elasticity theory", Acta Mechanica, 226, pp. 2277-2294 (2015).

12. Akgoz, B. and Civalek, O. "A novel microstructuredependent shear deformable beam model", International Journal of Mechanical Sciences, 99, pp. 10-20 (2015).
13. Li, A., Zhou, S., Zhou, S., and Wang, B.A. "Sizedependent model for bi-layered Kirchhoff micro-plate based on strain gradient elasticity theory", Composite Structures, 113, pp. 272-280 (2014).

14. Toupin, R.A. "Elastic materials with couple-stresses", Archive for Rational Mechanics and Analysis, 11, pp. 385-414 (1962).

15. Mindlin, R.D. and Tiersten, H.F. "Effects of couplestresses in linear elasticity", Archive for Rational Mechanics and Analysis, 11, pp. 415-448 (1962).

16. Yang, F., Chong, A.C.M., Lam, D.C.C., and Tong, P. "Couple stress based strain gradient theory for elasticity", International Journal of Solids and Structures, 39, pp. 2731-2743 (2002).

17. Jomehzadeh, E., Noori, H.R., and Saidi, A.R. "The size-dependent vibration analysis of micro-plates based on a modified couple stress theory", Physica E: LowDimensional Systems and Nanostructures, 43, pp. 877883 (2011).

18. Askari, A.R. and Tahani, M. "Analytical determination of size-dependent natural frequencies of fully clamped rectangular microplates based on the modified couple stress theory", Journal of Mechanical Science and Technology, 29, pp. 2135-2145 (2015).

19. Yin, L., Qian, Q., Wang, L., and Xia, W. "Vibration analysis of microscale plates based on modified couple stress theory", Acta Mechanica Solida Sinica, 23, pp. 386-393 (2010).

20. Asghari, M. "Geometrically nonlinear micro-plate formulation based on the modified couple stress theory", International Journal of Engineering Science, 51, pp. 292-309 (2012).

21. Gao, X.L, Huang, J.X, and Reddy, J.N. "A nonclassical third-order shear deformation plate model based on a modified couple stress theory", Acta Mechanica, 224, pp. 2699-2718 (2013).

22. Tsiatas, G.C. "A new Kirchhoff plate model based on a modified couple stress theory", International Journal of Solids and Structures, 46, pp. 2757-2764 (2009).

23. Shenas, A.G., Malekzadeh, P., and Mohebpour, S. "Vibrational behavior of variable section functionally graded microbeams carrying microparticles in thermal environment", Thin-Walled Structures, 108, pp. 122137 (2016).

24. Shenas, A.G., Ziaee, S., and Malekzadeh, P. "Vibrational behavior of rotating pre-twisted functionally graded microbeams in thermal environment", Composite Structures, 157, pp. 222-235 (2016).

25. Setoodeh, A.R. and Rezaei, M. "Large amplitude free vibration analysis of functionally graded nano/micro beams on nonlinear elastic foundation", Structural Engineering and Mechanics, 61, pp. 209-220 (2017). 
26. Ke, L.L. and Wang, Y.S. "Size effect on dynamic stability of functionally graded microbeams based on a modified couple stress theory", Composite Structures, 93, pp. 342-350 (2011).

27. Setoodeh, A.R. and Afrahim, S. "Nonlinear dynamic analysis of FG micro-pipes conveying fluid based on strain gradient theory", Composite Structures, 116, pp. 128-135 (2014).

28. Thai, H.T. and Kim, S.E. "A size-dependent functionally graded Reddy plate model based on a modified couple stress theory", Composites Part B: Engineering, 45, pp. 1636-1645 (2013).

29. Thai, H.T. and Choi, D.H. "Size-dependent functionally graded Kirchhoff and Mindlin plate models based on a modified couple stress theory", Composite Structures, 95, pp. 142-153 (2012).

30. Thai, H.T. and Vo, T.P. "A size-dependent functionally graded sinusoidal plate model based on a modified couple stress theory", Composite Structures, 96, pp. 376-383 (2013).

31. Ke, L.L., Yang, J., Kitipornchai, S., Bradford, M.A., and Wang, Y.S. "Axisymmetric nonlinear free vibration of size-dependent functionally graded annular microplates", Composites Part B: Engineering, 53, pp. 207-217 (2013).

32. Ansari, R., Shojaei, M.F., Mohammadi, V., Gholami, R., and Darabi, M.A. "Nonlinear vibrations of functionally graded Mindlin microplates based on the modified couple stress theory", Composite Structures, 114, pp. 124-134 (2014).

33. Lou, J. and He, L. "Closed-form solutions for nonlinear bending and free vibration of functionally graded microplates based on the modified couple stress theory", Composite Structures, 131, pp. 810-820 (2015).

34. Mohammadimehr, M. and Mohandes, M. "The effect of modified couple stress theory on buckling and vibration analysis of functionally graded double-layer Boron Nitride piezoelectric plate based on CPT", Journal of Solid Mechanics, 7, pp. 281-298 (2015).

35. He, L., Lou, J., Zhang, E., Wang, Y., and Bai, Y.A. "Size-dependent four variable refined plate model for functionally graded microplates based on modified couple stress theory", Composite Structures, 130, pp. 107-115 (2015).

36. Shenas, A.G. and Malekzadeh, P. "Free vibration of functionally graded quadrilateral microplates in thermal environment", Thin-Walled Structures, 106, pp. 294-315 (2016).

37. Roozbahani, M.M., Heydarzadeh Arani, N., Moghimi Zand, M., and Mousavi Mashhadi, M. "Analytical solutions to nonlinear oscillations of a microbeam using higher order beam theory", Scientia Iranica, 23(5), pp. 2179-2193 (2016).
38. Sedighi, H.M., Shirazi, K.H., and Zare, J. "An analytic solution of transversal oscillation of quintic non-linear beam with homotopy analysis method", International Journal of Non-Linear Mechanics, 47, pp. 777-784 (2012).

39. Alipour, A., Zand, M.M., and Daneshpajooh, H. "Analytical solution to nonlinear behavior of electrostatically actuated nanobeams incorporating van der Waals and Casimir forces", Scientia Iranica, 22(3), pp. 1322-1329 (2015).

40. Liao, S.J. "Advances in the homotopy analysis method", World Scientific, New York (2013).

41. Rao, G.V., Raju, I.S., and Raju, K.K. "A finite element formulation for large amplitude flexural vibrations of thin rectangular plates", Computers \& Structures, 6, pp. 163-167 (1976).

42. Ventsel, E. and Krauthammer, T. "Thin plates and shells: theory: analysis, and applications", CRC Press, New York (2001).

43. Mindlin, R.D. "Influence of rotary inertia and shear on flexural motions of isotropic elastic plates", Journal of Applied Mechanics, 18(1), pp. 31-38 (1951).

44. Christoforou, A.P. and Swanson, S.R. "Analysis of simply-supported orthotropic cylindrical shells subject to lateral impact loads", Journal of Applied Mechanics, 57(2), pp. 376-382 (1990).

45. Rao, S.S., Vibration of Continuous Systems, John Wiley \& Sons, New Jersey (2007).

46. Liao, S.J., Beyond Perturbation: Introduction to Homotopy Analysis Method, CRC press, Boca Raton (2004).

47. Han, W. and Petyt, M. "Geometrically nonlinear vibration analysis of thin, rectangular plates using the hierarchical finite element method 2014 I: the fundamental mode of isotropic plates", Computers \& Structures, 63, pp. 295-308 (1997).

48. Sundararajan, N., Prakash, T., and Ganapathi, M. "Nonlinear free flexural vibrations of functionally graded rectangular and skew plates under thermal environments", Finite Elements in Analysis and Design, 42, pp. 152-168 (2005).

\section{Appendix}

The coefficients of Eq. (31) are defined in Box A.I, where functions $X(x)$ and $Y(y)$ are, respectively, abbreviated by $X$ and $Y$. Also, the subscript comma denotes a partial derivative with respect to the corresponding coordinate:

$$
\begin{aligned}
& \Delta_{1}=C_{2}+\frac{A_{1} l^{2}}{2}, \\
& \Delta_{2}=2 C_{1}+2 C_{3}+A_{1} l^{2}, \\
& \Delta_{3}=B_{1}-2 B_{3}, \\
& \Delta_{4}=-\frac{A_{1}+A_{3}}{2},
\end{aligned}
$$




$$
\begin{aligned}
\bar{\alpha}= & \frac{\int_{0}^{a} \int_{0}^{b}\left\{X Y\left[\Delta_{1}\left(X_{, x x x} Y+X Y_{, y y y y}\right)+\Delta_{2}\left(X_{, x x} Y_{, y y}\right)\right]\right\} d x d y}{\Delta_{5}}, \\
\bar{\beta}= & \frac{\int_{0}^{a} \int_{0}^{b}\left\{X Y\left[\Delta_{3}\left(\left(X_{, x} Y_{, y}\right)^{2}-X Y X_{, x x} Y_{, y y}\right)\right]\right\} d x d y}{\Delta_{5}} \\
\bar{\gamma}= & \frac{\int_{0}^{a} \int_{0}^{b}\left\{X Y\left[-1.5 A_{2}\left(X_{, x x} Y\left(X_{, x} Y\right)^{2}+X Y_{, y y}\left(X Y_{, y}\right)^{2}\right)\right]\right\} d x d y}{\Delta_{5}} \\
& +\frac{\int_{0}^{a} \int_{0}^{b}\left\{X Y\left[\Delta_{4}\left(\left(X Y_{, y}\right)^{2} X_{, x x} Y+(X, x Y)^{2} X Y_{, y y}\right)+4 \Delta_{4}\left(X Y\left(X_{, x}\right)^{2}\left(Y_{, y}\right)^{2}\right)\right]\right\} d x d y}{\Delta_{5}} .
\end{aligned}
$$

\section{Box A.I}

$$
\Delta_{5}=\int_{0}^{a} \int_{0}^{b}\left[X Y\left(I_{0} X Y-I_{2} X_{, x x} Y-I_{2} X Y_{, y y}\right)\right] d x d y
$$

\section{Biographies}

Ali Reza Setoodeh is a Professor in the Department of Mechanical and Aerospace Engineering, Shiraz University of Technology, Iran. He obtained his BSc, MSc and $\mathrm{PhD}$ degree in Mechanical Engineering, from the Shiraz University, Iran. His research interests are com- putational mechanics, computational nano-mechanics, modeling, and fabrication of nano-composite materials and machine design.

Mohammad Rezaei received his BSc degree in Mechanical Engineering from Shahid Chamran University of Ahvaz, Ahvaz, Iran, in 2009. He also received his MSc degree in Mechanical Engineering from Shiraz University of Technology, Iran, in 2013. His research interests include functionally graded materials, and micro/nano-mechanics. 